Textures and Microstructures, Vol. 33, pp. 75-92 Reprints available directly from the publisher Photocopying permitted by license only
(C) 1999 OPA (Overseas Publishers Association) N.V. Published by license under the Gordon and Breach Science Publishers imprint. Printed in Malaysia.

\title{
NEUTRON TEXTURE ANALYSIS OF MELT-TEXTURED YBCO BULK SAMPLES
}

\author{
LOTHAR SCHMIDT $^{\mathrm{a}, *}$, MARTIN ULLRICH ${ }^{\mathrm{b}}$ \\ and WERNER F. KUHS ${ }^{\mathrm{a}}$

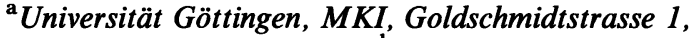 \\ D-37077 Göttingen, Germany; ${ }^{\mathrm{b}} \mathrm{ZFW}$ Göttingen gGmbH, \\ Windausweg 2, D-37073 Göttingen, Germany
}

\begin{abstract}
Neutron texture measurements on YBCO bulk samples show a very sharp texture of the superconducting phase $\mathrm{YBa}_{2} \mathrm{Cu}_{3} \mathrm{O}_{7-x}$ with half-widths of less than $5^{\circ}$. Even with a rather coarse measurement grid of only 722 points per complete pole figure, satisfactory results for the recalculated (002) pole figures could be obtained. However, for a reliable calculation of a complete ODF, finer grids will have to be used. Due to the importance of a good alignment of the $c$-axes in the material, a quantitative analysis of the (002) pole figures, including an error estimation due to measurement grid and counting statistics, was made. An outline for the determination of a reliable background estimate is given.
\end{abstract}

Keywords: Texture; YBCO; High temperature superconductors

\section{INTRODUCTION}

Bulk samples of the superconducting ceramic phase $\mathrm{YBa}_{2} \mathrm{Cu}_{3} \mathrm{O}_{7-x}$ (orthorhombic, $a=3.821, b=3.888, c=11.693$ for $x=0.2$ (JCPDSICDD, 1996)) with other phase additions (e.g. $\mathrm{Y}_{2} \mathrm{O}_{3}, \mathrm{Y}_{2} \mathrm{BaCuO}_{5}, \mathrm{PtO}_{2}$, $\mathrm{CeO}_{2}$ ) are potentially useful in magnetic applications. The levitation force between such an YBCO sample and a permanent magnet depends on what is referred to as the "domain structure" of $\mathrm{YBa}_{2} \mathrm{Cu}_{3} \mathrm{O}_{7-x}$ (Görnert, 1997). A domain in this sense is a part of the sample where superconducting currents can flow without being hindered e.g. by cracks, oxygen-deficient zones or large-angle grain boundaries. Each domain

\footnotetext{
* Corresponding author.
} 
can be characterised by scanning the remnant magnetic field with a Hall probe. A domain may be smaller than a crystallographic grain.

While Hall-probe scans along a sample surface immediately measure the remnant magnetic field with good local resolution (cf. Görnert, 1997), neutron texture analysis has the drawback of not providing any information about the spatial distribution of the potential domains in the sample. Neither can it detect intragranular cracks. It has, however, the great advantage of being a non-destructive means to analyse the whole (or at least a large part) of the bulk. With the total absorption cross sections given in the literature (Sears, 1992), the penetration depth of thermal neutrons in $\mathrm{YBa}_{2} \mathrm{Cu}_{3} \mathrm{O}_{7-x}$ is found to be greater than $3 \mathrm{~cm}$ at $\lambda=1.344 \AA$, which is more than three orders of magnitude larger than for X-rays at the $\mathrm{Cu}_{\mathrm{K} \alpha}$ wavelength. The Hall-probe method (Frangi et al., 1994) has a penetration depth of the order of a few $\mathrm{mm}$.

Although neutron texture analysis cannot reveal the actual domain structure, it allows to make hypotheses about the grain structure. Melttextured YBCO samples frequently have grains with diameters in the cm scale (cf. Görnert, 1997; Marinel et al., 1997). In terms of texture analysis, the orientations of these grains are visible as strong texture components. Since grain boundaries with a misorientation angle greater than $10^{\circ}$ always act as domain boundaries (Dimos et al., 1988), an upper limit of the sizes as well as a lower limit of the number of domains in the sample can be determined by quantitative neutron texture analysis.

\section{EXPERIMENTAL}

All experiments were made on the TEX2-beamline of the GKSS Forschungszentrum $\mathrm{GmbH}$ with a neutron wavelength of $\lambda=1.344 \AA$, using a primary $\mathrm{Cu}-(111)$ monochromator. Data collection time was controlled by monitor counts. Diffracted neutrons were registrated by a $\mathrm{He}^{3}$ single detector with a $20 \times 20 \mathrm{~mm}^{2}$ aperture. The initial primary neutron beam divergence of $52^{\prime}$ was additionally reduced by a Cd mask that limited the primary beam diameter to $22 \mathrm{~mm}$. The sample-detector distance was $120 \mathrm{~cm}$ for most of the measurements.

Two of the samples, named " $\mathrm{fb} 12$ " and " $\mathrm{m} 209$ ", were cylinders of $\oslash$ $31 \mathrm{~mm}$, height $15 \mathrm{~mm}$. A third sample, "hzj", was a cylinder of 
approximately the same dimensions that had been cut in two halves along its axis.

For most of the results presented here, a data collection grid consisting of 679 points per pole figure was used. The number of grid points per complete pole figure would be 722 since only half of the $\beta$ circle was measured at $\alpha=90^{\circ}$. Hence, this grid will be referred to as the 722 points grid. The solid angle attributed to each grid point was constant over the pole figure hemisphere, and the mean distance between neighbouring points was $5.62^{\circ}$. For all samples, at least two pole figures were measured. The first one was the (002) pole figure, which shows no superposition of other Bragg reflections of the first order of the monochromator. The multiplicity of the (002) poles being only two, the number of distinct (002) pole density maxima is in direct correspondence with the minimum number of differently oriented domains in the sample. Thus, a high concentration of (002) pole density is necessary for "good" YBCO bulk samples. The second one was the superposed $(005)+(014)+(104)$ pole figure: It reflects once more the orientations of the $c$-axes, and the (014) and (104) pole density distributions can indirectly reveal the orientation of the $a$ - and the $b$-axes. The reflections are relatively strong. A significant difference in the squared structure factors of the (014) and the (104) reflections exists, the former being stronger by a factor of about 2.5. Moreover, further pole figures were measured, such as the superposed $(012)$ and $(102)$ or $(013)+(103)+(110)$ pole figures, or the (113) pole figure. There is also a squared structure factor difference of a factor of roughly two between (013) and (103).

\section{RECALCULATION METHOD}

The iterative series expansion method proposed by Dahms and Bunge (1989) was used to obtain recalculated pole figures as well as the $C$ coefficients of the orientation distribution function (ODF). This method allows to use a comparatively high series expansion degree even with rather few experimental pole figures. In the results presented here, the maximum expansion degree was $l_{\max }=34$, using three or four experimental pole figures. Orthorhombic crystal and triclinic sample symmetries were assumed. The method also allows to include overlapped pole figures when information about the relative powder 
intensities of the overlapping reflections is supplied. A maximum number of three reflections per pole figure could be treated here.

\section{RESULTS}

The agreement between the measured and the recalculated pole figures was quite satisfactory, with a range of $\mathrm{RP}_{1}$ values between below four and 14. A strong preferred orientation was found in all of the samples, the texture indices being 311,145 and 71 for the samples "hzj", " $\mathrm{m} 209$ " and "fb12" (for a definition of the texture index see e.g. Bunge, 1969).

In the plots, the angle denoted by "Phi" is the angle commonly referred to as " $\beta$ " (see Bunge, 1986). For the angle " $\alpha$ ", the goniometer angle $\chi$ will be used synonymously. The solid circles in the pole figure plots are at $\chi=30^{\circ}$ and $\chi=60^{\circ}$.

Figure 1(a) shows the experimental pole figures (002) (left) and $(005)+(014)+(104)$ (right) of the sample "hzj". In Fig. 1(b), the latter has been decomposed into its three constituents by the iterative series expansion method. The strong pole density maximum near the centre is attributed mainly to the (005) reflection, but also to the (014) reflection. Correspondingly, in the recalculated (002) i.e. (005) pole figure, there is a slightly increased pole density at the positions of the (014) and (104) maxima which has no correspondence in Fig. 1(a). Thus, the nearly central maximum in the recalculated (014) pole figure is probably an artefact of the recalculation which may have its origin in slightly wrong superposition factors for (014) and (005).

(a)

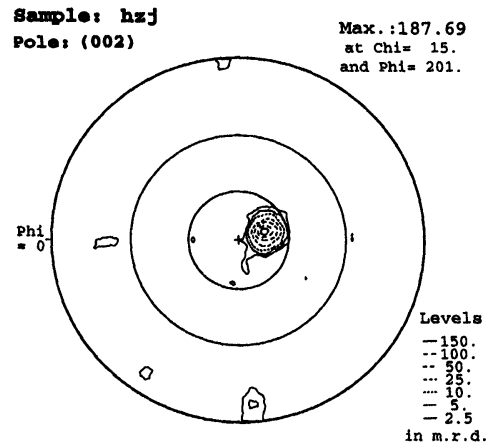

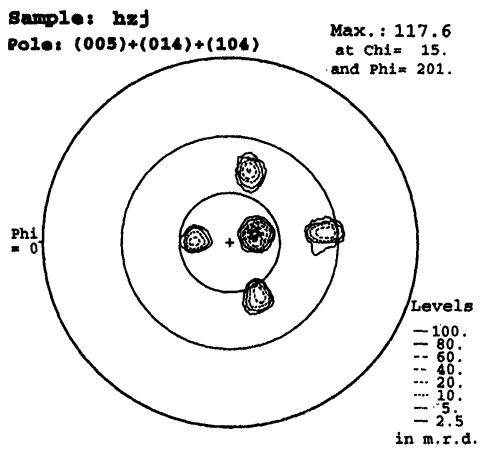

FIGURE 1(a) 
(b)

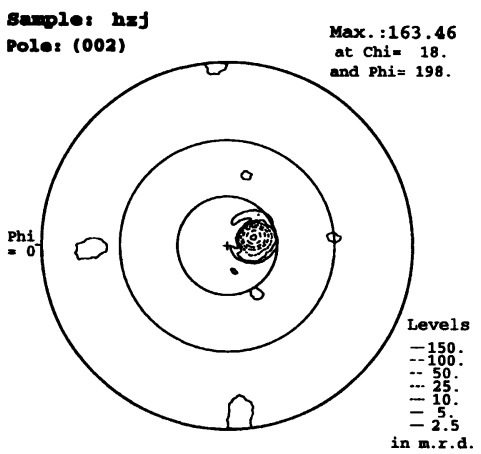

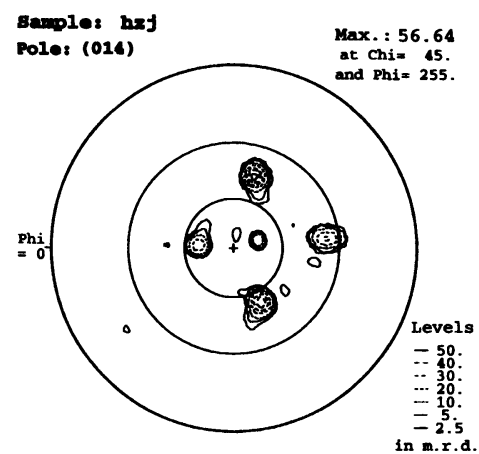

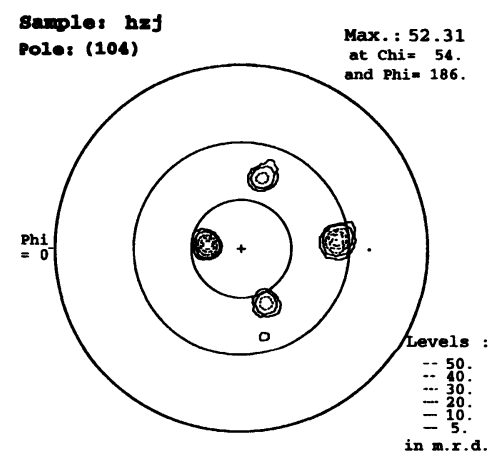

FIGURE 1(b)

FIGURE 1 (a) Experimental pole figures for sample "hzj". Left: (002). Right: $(005)+(014)+(104)$. (b) Sample "hzj". Decomposition of the $(005)+(014)+(104)$ pole figure. Top left: Recalculated (002); Top right: Recalculated (014); Bottom: Recalculated (104).

Another feature of the texture is the overlap of the recalculated (014) poles with the (104) poles. The overlap of these two poles shows that there are two texture components which have a common $c$-axis orientation but differ from each other by a $90^{\circ}$-rotation around (001). This will be a result of the formation of twin lamellae along planes of the (110) type, which is frequently observed in the material (Diko et al., 1996). However, Fig. 1(b) also shows that preferentially the (104) poles seem to form the maxima at $\varphi=0^{\circ}$ and $\varphi=180^{\circ}$, and the (014) poles those at $\varphi=90^{\circ}$ and $\varphi=270^{\circ}$. Apparently, there is no complete symmetry concerning the distribution of the $a$ - and the $b$-axes in the texture. 
The most conspicuous $\varphi$-dependence of the intensities of individual maxima is visible in the superposed $(013)+(103)+(110)$ pole figure (see Fig. 2). However, as will be discussed below, this is more likely to be an effect of a too coarse measurement grid.

The samples "hzj" and " $\mathrm{m} 209$ " have been reexamined in a later experimental period, using more accurate data collection grids, i.e. $2.5^{\circ} \times 2.5^{\circ}$ for sample "hzj" and $2^{\circ} \times 2^{\circ}$ for sample "m209". For reasons of limited allocated time, the measured areas were restricted to the maxima of the $(013)+(103)$ intensity distribution (these poles can be distinguished from the (110) poles with the help of the (002) pole figure) as well as to those of (014) $+(104)$ and (005). The aim was to determine the half-widths and intensities of these maxima with greater accuracy.

For the sample "hzj", Fig. 2 shows new results for the (013) and (103) maxima (left). On the right side of Fig. 2, the $(013)+(013)+(110)$ pole figure measured with the 722 points grid is shown. In the latter, there is an obvious difference in the intensities between the maxima at $\varphi=0^{\circ} \%$ $\varphi=180^{\circ}$ and those at $\varphi=90^{\circ} / \varphi=270^{\circ}$. In the former, no such difference is apparent. Also, the integrated intensities of the four individual maxima have been calculated. For the measurement with the 722 points grid, they were found to be about $25 \%$ lower at $\varphi=90^{\circ}$ and $\varphi=270^{\circ}$ than at $\varphi=0^{\circ}$ and $\varphi=180^{\circ}$. For the finer grid, they still vary about $10 \%$, but do not show the same systematic dependence on $\varphi$.

Neither is there a $\varphi$-dependent periodicity of the integrated intensities of the non-(005) poles in the superposed $(014)+(104)+(005)$ pole
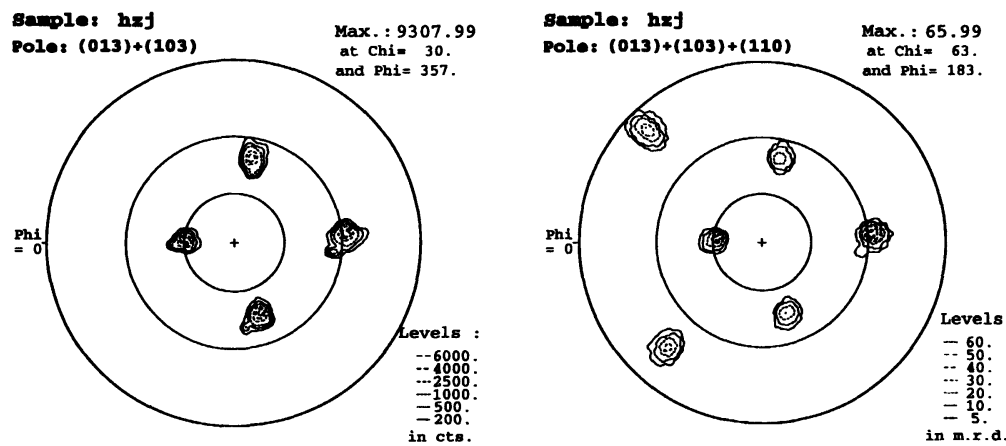

FIGURE 2 Sample "hzj". Left: Maxima of $(013)+(013)$ pole distribution measured with a $2.5^{\circ} \times 2.5^{\circ}$ grid. Right: $(013)+(013)+(110)$ pole figure measured with the 722 points per complete pole figure grid (intensities have been normalised to multiples of random distribution (m.r.d.)). 
figure, neither for the more accurate data nor for those measured with the 722 points grid. It must then be concluded that the result of the recalculation based on the latter was strongly influenced by the apparent periodicity in the intensity distribution of the superposed (013)+ $(013)+(110)$ pole figure.

But, as the results obtained with the finer grid show, this is rather an effect of a too coarse data collection grid that must have missed the peaks of some of the (013) + (103) maxima and not a real feature of the texture of this sample. Further below, the effect of a too coarse measurement grid will be quantitatively discussed.

For the $(013)+(103)$ maxima of the sample "m209", the results obtained with the finer grid are shown in Fig. 3. For the individual maxima, half-widths in $\Delta \chi$ and in $\sin (\chi) \Delta \varphi$ have been determined. The average values are shown in Table I. These values were determined

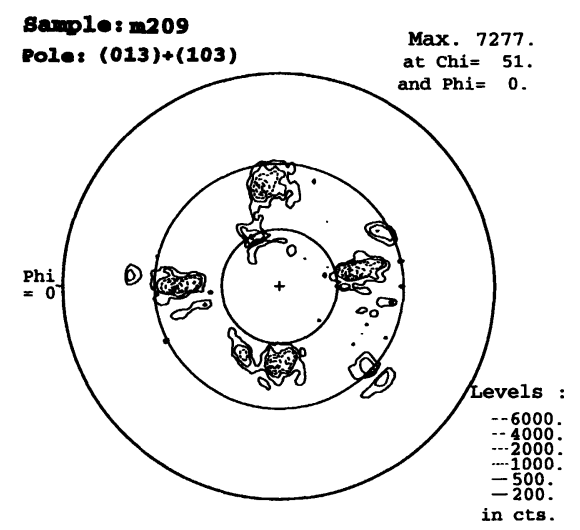

FIGURE 3 Sample "m209". (013)+(013) intensity distribution measured with a $2^{\circ} \times 2^{\circ}$ grid.

TABLE I Full widths at half maximum values obtained with a $2.5^{\circ} \times 2.5^{\circ}$ (sample "hzj") resp. $2^{\circ} \times 2^{\circ}$ (sample "m209") measurement grid. The values are the average of $\Delta \chi$ and $\sin (\chi) \Delta \varphi$. For $(014)+(104)$ and $(013)+(103)$, the average values of the four maxima are shown

\begin{tabular}{lcc}
\hline Poles & $h z j$ & $m 209$ \\
\hline$(005)$ & $5.1^{\circ}$ & $3.8^{\circ}$ \\
$(014)+(104)$ & $4.6^{\circ}$ & $4.0^{\circ}$ \\
$(013)+(103)$ & $4.7^{\circ}$ & $4.7^{\circ}$ \\
\hline
\end{tabular}


graphically from more detailed plots. Describing the maxima in terms of half-widths presupposes that they are single peaks which can be approximated by Gaussian bell curves. For some of the more scattered maxima, e.g. those for (013) +(103) in the sample " $\mathrm{m} 209$ ", this is not strictly the case (cf. Fig. 3). The discrepancy between the half-widths for $(013)+(013)$ and $(014)+(104)$ in the sample "m209" can also have its origin in the $2 \theta$-dependence of the instrumental resolution for the angle $\chi$. The $(013)+(103)$ reflection was measured at $2 \theta=28.4^{\circ}$ and $(014)+(104)$ at $2 \theta=33.4^{\circ}$. The instrumental resolution for $\chi$ is found to improve with $2 \theta$, which can explain why the half-width $\Delta \chi$ is larger for $(013)+(103)$ than for $(014)+(104)$, and thus leads to the higher average value.

\section{A Quantitative Evaluation of Texture Components with a Common $c$-axis Orientation}

Given the importance of a good alignment of the crystallographic $c$-axes (Jin et al., 1988), a quantitative analysis of the (002) orientation distribution has been made. In the experimental (002) pole figures of the samples "hzj", "m209" and "fb12", as shown in Fig. 4, solid angle areas comprising the main maxima have been marked by dashed lines.

The volume fractions of texture components having a $c$-axis orientation within one of these marked areas, denoted by " $v_{i}$ ", can be calculated as follows (cf. Bunge, 1986):

$$
v_{i}=\frac{1}{2 \pi} \int_{\Omega_{i}} P_{\vec{h}}(\vec{y}) \mathrm{d} \vec{y} .
$$

Here, $\vec{h}=(002) . P_{\vec{h}}(\vec{y})$ is then the (002) pole density normalised over the measured hemisphere (thus, the normalisation factor in Eq. (1) is $(1 / 2 \pi)$ instead of $(1 / 4 \pi)$ as in (Bunge, 1986)). $\Omega_{i}$ is the marked solid angle area in the (002) pole figure, $\vec{y}$ is a direction in the sample, given by the two pole figure angles $(\alpha, \beta)$, and $\mathrm{d} \vec{y}$ is a solid angle element around $\vec{y}$.

When the data are collected at discrete points, Eq. (1) is approximated by

$$
v_{i}=\frac{1}{2 \pi} \sum_{j}^{\Omega_{i}} P_{\vec{h}}\left(\vec{y}_{j}\right) \mathrm{d} \vec{y}_{j} .
$$



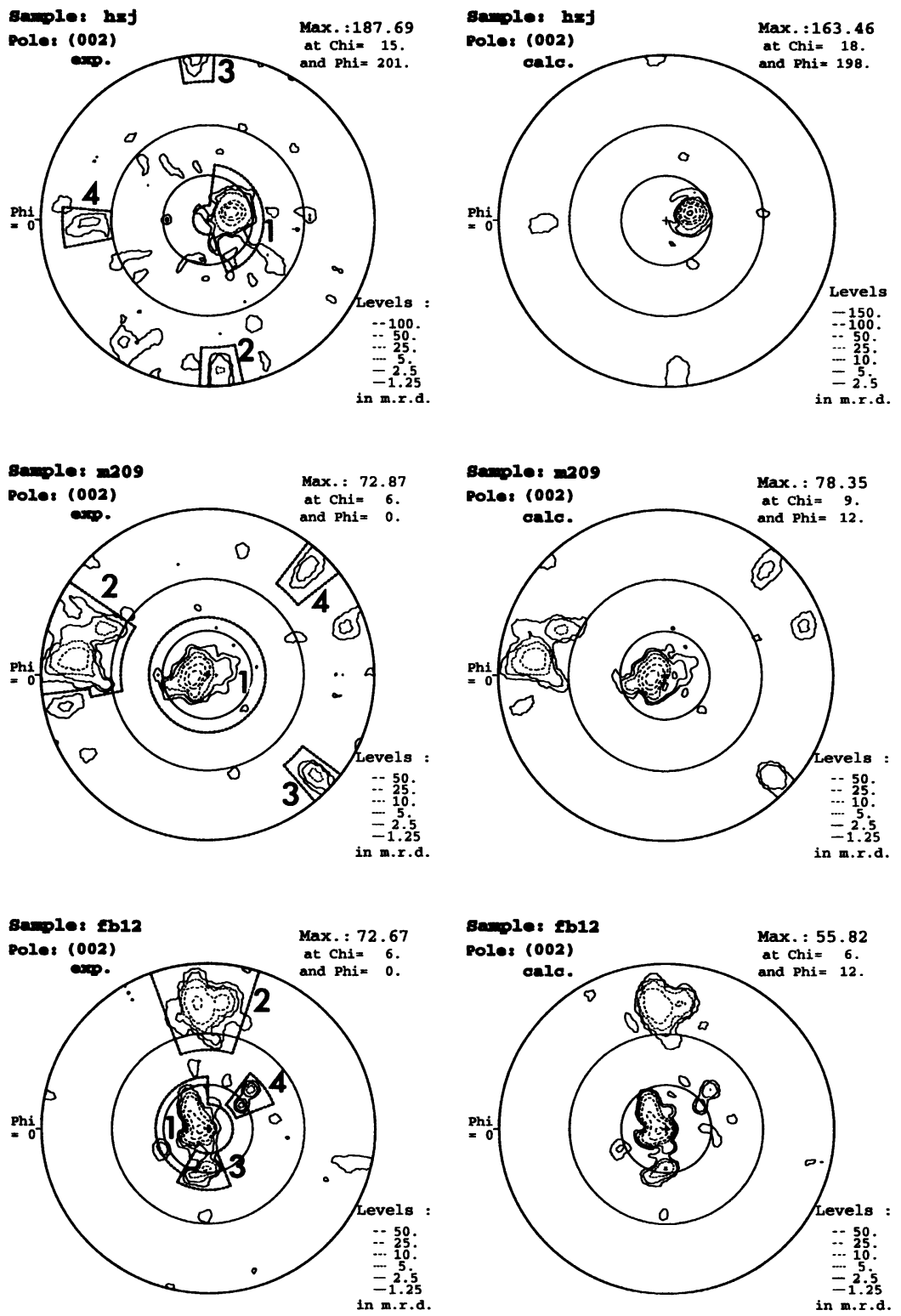

FIGURE 4 Experimental (left) and recalculated (right) (002) pole figures. Solid angle areas where volume fractions are to be calculated have been marked in the experimental pole figures. 
The sum is over all grid points lying within the area and $\Omega_{i}$ and $\mathrm{d} \vec{y}_{j}$ is the element of solid angle attributed to the grid point " $j$ ". The pole density $\boldsymbol{P}_{\vec{h}}\left(\vec{y}_{j}\right)$ is then given by

$$
P_{\vec{h}}\left(\vec{y}_{j}\right)=2 \pi\left(I\left(\vec{y}_{j}\right)-\mathrm{BK}\right) / \sum_{k}^{2 \pi}\left(I\left(\vec{y}_{k}\right)-\mathrm{BK}\right) \mathrm{d} \vec{y}_{k},
$$

where $I\left(\vec{y}_{j}\right)$ is the total intensity measured at grid point " $\vec{y}$ ". BK is the background intensity, which is assumed to be constant all over the pole figure. A geometrical consideration revealed that due to the low absorption of thermal neutrons in our material, there was no necessity to correct the measured intensities for effects like absorption or secondary beam broadening at the sample and instrument geometries used here.

The volume fractions of the solid angle areas marked in Fig. 4 are shown in Table II. The same area limits were used for the recalculated pole figures. All values are given in percent of irradiated sample volume.

From Eqs. (2) and (3), an error analysis can be attempted. Its results were denoted by " $\sigma$ " in Table II. Two effects were taken into account: (a) the coarseness of the 722 points measurement grid leading to wrong values of the integrated intensities, and (b) the error due to counting statistics. The two aspects are considered as uncorrelated, and the resulting total $\sigma$ is then given by $\left(\sigma_{\text {grid }}^{2}+\sigma_{\text {count }}^{2}\right)^{1 / 2}$. The individual contributions of (a) and (b) are shown in Table III.

For the quantitative estimation of (a), a simulation method was used. It was assumed that the intensity data shown in Fig. 2 (left) and in Fig. 3 for $(013)+(013)$ were measured with the best possible grid resolution. Measurements using the 722 points grid were then simulated by

TABLE II Volume fractions of the solid angle areas assigned in Fig. 4 for both experimental and recalculated (002) pole figures. Values are given in percent of irradiated sample volume. Sigma is an estimated standard deviation, as explained in the text

\begin{tabular}{|c|c|c|c|c|c|c|c|c|c|}
\hline \multirow{2}{*}{$\begin{array}{l}\text { Sample } \\
\text { area no. }\end{array}$} & \multicolumn{3}{|c|}{$h z j$} & \multicolumn{3}{|c|}{$m 209$} & \multicolumn{3}{|c|}{$f b 12$} \\
\hline & exp. & calc. & $\sigma$ & exp. & calc. & $\sigma$ & exp. & calc. & $\sigma$ \\
\hline "1" & 94.9 & 89.3 & 2.5 & 74.2 & 74.6 & 3.7 & 61.0 & 58.6 & 3.9 \\
\hline “2”" & 1.6 & 2.1 & 0.9 & 21.1 & 17.0 & 3.0 & 27.9 & 22.4 & 3.4 \\
\hline "3" & 0.6 & 0.8 & 0.7 & 2.3 & 2.0 & 0.6 & 7.1 & 7.1 & 1.2 \\
\hline “4" & 1.8 & 2.9 & 1.0 & 2.0 & 1.6 & 0.6 & 3.3 & 3.0 & 0.8 \\
\hline Sum: & 98.9 & 95.1 & & 99.6 & 95.2 & & 99.3 & 91.1 & \\
\hline
\end{tabular}


TABLE III Contributions of counting statistics and measurement grid to the total expected error denoted by "sigma" in Table II. All values are given in percent of irradiated sample volume

\begin{tabular}{|c|c|c|c|c|c|c|}
\hline \multirow{2}{*}{$\begin{array}{l}\text { Sample } \\
\text { area no. }\end{array}$} & \multicolumn{2}{|c|}{$h z j$} & \multicolumn{2}{|c|}{$m 209$} & \multicolumn{2}{|c|}{$f b 12$} \\
\hline & $\sigma_{\text {grid }}$ & $\sigma_{\text {count }}$ & $\sigma_{\text {grid }}$ & $\sigma_{\text {count }}$ & $\sigma_{\text {grid }}$ & $\sigma_{\text {count }}$ \\
\hline "1" & 0.7 & 2.4 & 3.0 & 2.0 & 3.6 & 1.4 \\
\hline “2” & 0.3 & 0.9 & 2.8 & 1.2 & 3.2 & 1.0 \\
\hline “3”" & 0.1 & 0.7 & 0.3 & 0.4 & 1.0 & 0.7 \\
\hline "4" & 0.3 & 1.0 & 0.3 & 0.5 & 0.5 & 0.6 \\
\hline
\end{tabular}

interpolating these data at the positions of the 722 grid points. A total of 4000 simulation cycles per pole figure was run. For each cycle, a relative orientation of sample and grid was determined by three Eulerian angles $\left(\varphi_{1}, \phi, \varphi_{2}\right)$ chosen randomly within the intervals $\left[0^{\circ}, 360^{\circ}\right]$ for $\varphi_{1}$, $\left[0^{\circ}, 2.5^{\circ}\right]$ for $\phi$ and $\left[0^{\circ}, 360^{\circ}\right]$ for $\varphi_{2}$. This was meant to represent the arbitrariness of the $\beta=0^{\circ}$-position as well as a possible slight misalignment of the cylinder axis for each of the simulated measurements. As a result, it was found that the expected relative error (square root of variance divided by mean value) of the integrated intensities of the individual maxima was of the order of $10 \%$. The same procedure was applied to the $(014)+(104)$ and the $(005)$ maxima of both samples, leading to a similar value.

Due to the decrease in instrumental resolution at lower $2 \theta$ values, the relative error for a (002) maximum, measured at $2 \theta=13.2^{\circ}$, can be expected to be somewhat smaller than, e.g., for a (005) maximum appearing at $2 \theta=33.4^{\circ}$. On new data, half-widths in $\Delta \chi$ were found to increase by a factor of 1.3 for (002) with respect to (005). Nevertheless, for the estimation of $\sigma_{\text {grid }}$, a relative error of $10 \%$ of the integrated (002) intensities will be assumed.

In order to apply this result to the calculated volume fractions, it was assumed that only in a few distinct areas of the (002) pole figure, like those marked in Fig. 4, the pole density is greater than zero. The sum of the volume fractions attributed to these areas will then be one. Each of these areas should contain exactly one maximum. All maxima should have equal half-widths, so that the effect of a coarse measurement grid is the same for all of them. In practice, these conditions will not always be strictly fulfilled (cf. Fig. 4). Thus, it has to be stressed here that the $\sigma_{\text {grid }}$ values shown in Table III should be taken as estimates, and not as exact figures. 
When, due to a coarse measurement grid, the relative error of the integrated intensity of each of the solid angle areas in a (002) pole figure is $10 \%$, the inaccuracy thus introduced on the volume fraction " $v_{i}$ " attributed to area No. " $i$ " is (cf. Appendix):

$$
\sigma_{\text {grid }}\left(v_{i}\right)=10 \% v_{i}\left(\left(1-v_{i}\right)^{2}+\sum_{j \neq i}^{\# \text { Other Areas }} v_{j}^{2}\right)^{1 / 2} .
$$

In order to estimate the effect of (b), i.e. the counting statistics, as a source of error for the determination of volume fractions, all the measured intensities were taken as uncorrelated variables. Also, the background intensity at each grid point was considered as an independent variable. The error-propagation rule was then applied to Eq. (2).

\section{The Influence of an Inaccurately Determined Mean Background Value}

The crucial importance of an accurate knowledge of the mean background intensity (assumed to be constant all over the pole figure) can be seen by inserting Eq. (3) into Eq. (2) and then deriving Eq. (2) with respect to BK, i.e. the mean background intensity (cf. Appendix). The following quantitative relationship is also illustrated in Fig. 5:

$$
\frac{\Delta v_{j}}{v_{i}}=\frac{\Delta \mathrm{BK}}{\mathrm{BK}} \cdot 1 /\left(\frac{\langle I\rangle}{\mathrm{BK}}-1\right) \cdot\left(1-\frac{\Omega_{i}}{2 \pi v_{i}}\right) .
$$

Equation (5) is approximately valid only for small values of $\Delta B K / B K$, where the change of $\mathrm{BK}$ in the denominator can be neglected. It shows the effect of a shift in the background estimate, denoted by $\Delta \mathrm{BK}$, on the volume fraction attributed to the area No. " $i$ ", $v_{i}$. As in Eq. (2), $\Omega_{i}$ is the solid angle over which the intensity was integrated., i.e. $\Omega_{i} / 2 \pi$ is the fraction of the pole figure hemisphere occupied by the maximum in question. $\langle I\rangle$ is the mean value of intensity measured on the pole figure, including the background. As Fig. 5 illustrates, the change of $v_{i}$ is always to the negative when $\triangle \mathrm{BK} / \mathrm{BK}$ is negative, i.e. when the background is estimated too low. In this case, the excess of intensity that remains in a (002) pole figure after the background correction will be interpreted as a texture component with randomly distributed $c$-axis. Sharp maxima 

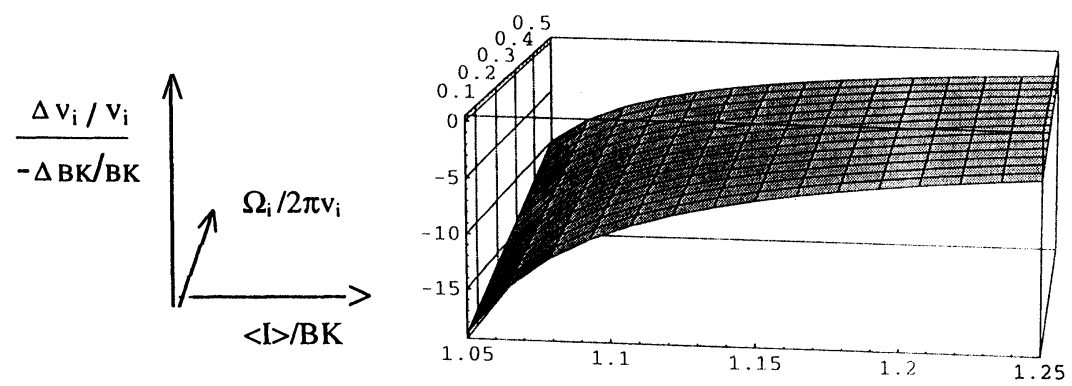

FIGURE 5 Effect of a shift in the background estimate $(\Delta \mathrm{BK})$ on the volume fraction $v_{i}$. Sharp maxima (low values of $\Omega_{i} / 2 \pi v_{i}$ ) are more sensitive to shifts of the assumed background than diffuse maxima. Weak Bragg reflections measured with a high background will have a $\langle I\rangle / \mathrm{BK}$ ratio only slightly greater than one and thus require a very accurate determination of the background.

with high pole densities covering a small solid angle are more sensitive to a background misestimation than diffuse maxima. Also, weak Bragg reflections measured with a high background will have a $\langle I\rangle / \mathrm{BK}$ ratio only slightly greater than one and thus require a very accurate determination of the background. Although our (002) pole figures are good examples for the latter case, the effect of a possible misestimation of the mean background could not be included in the quantitative error considerations in Table II because of the difficulty to guess the inaccuracy of our mean background estimate.

The determination of the background intensity is not a trivial task in a material such as $\mathrm{YBCO}$, where many reflections of the main phase $\mathrm{YBa}_{2} \mathrm{Cu}_{3} \mathrm{O}_{7-x}$ ("YBCO-123") overlap and where a second phase, $\mathrm{Y}_{2} \mathrm{BaCuO}_{5}$ ("YBCO-211"), is also present. In melt-textured material, the weight percentage of $\mathrm{YBCO}-211$ is usually of the order of about $30 \%$. If a multiphase texture analysis is not intended or not possible, an overlap of a reflection of the 211-phase with a reflection of the 123-phase will increase the background in the pole figure of the latter, provided there is no strong texture of the 211-phase (recent publications (Chateigner et al., 1997; Endo and Shiohara, 1997) hint to a preferred orientation of the 211phase, qualified as "weak" by Chateigner et al. (1997); so this point will have to be investigated). A background value taken at a two-theta angle somewhat off the Bragg angle will then be too small.

A possible way out of this problem is to take the background value directly from the measured pole figure data. This can be done with the 
help of a Poisson distribution fit, which works well when there are only a few and well-defined maxima in the pole figure, as it is the case for the (002) pole figure of the sample "hzj" (see Fig. 6).

If there are more maxima present, if different Bragg reflections overlap in one pole figure, or if the texture is smoother, a method has to be devised to carry out the statistics only over the points of the pole figure where there is no Bragg contribution of the 123-phase. These data points have then to be sorted out, which can be done by assuming that the maxima are coherent in terms of solid angle and can thus be "cut out" from the data set.

However, it should be noticed that trying to find the best approximation for the background by this method cannot distinguish between actual background and a texture component of random orientation, i.e. a so-called "fon", of the 123-phase. This is why separate background measurements should be done in any case. To decide whether or not there might be a "fon" component in the 123-texture, the measured background values and the result of the poisson fit for every pole figure can be compared. If the latter is always greater than the former and if the difference is always in correlation with the structure factors of the respective Bragg reflections, then that can be a hint to the existence of a "fon" component in the 123-texture.

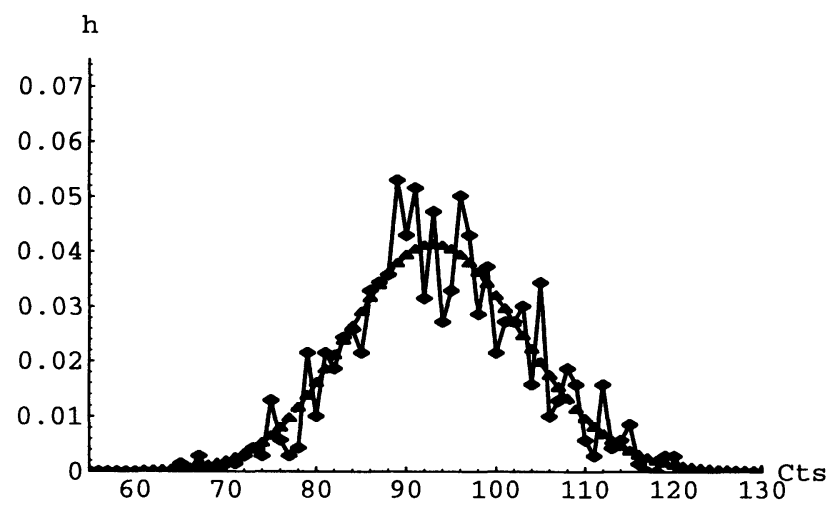

FIGURE 6 Poisson distribution fit over counting rates in the experimental (002) pole figure of sample "hzj", where the maxima have been excluded. The vertical axis is scaled in terms of probability. Empiric values are denoted by rhombae, fitted values by triangles. 


\section{CONCLUSION}

Very sharp textures in terms of half-widths as well as of texture index were found in melt-textured $\mathrm{YBa}_{2} \mathrm{Cu}_{3} \mathrm{O}_{7-x}$ bulk samples of cylindrical shape with a typical diameter of $31 \mathrm{~mm}$.

The half-widths, which were found to be of less than $5^{\circ}$ for the sharpest maxima, were measured with $2^{\circ} \times 2^{\circ}$ or $2.5^{\circ} \times 2.5^{\circ}$ equal-angular data collection grids. Pole figures that have been used as input data for ODF calculations were measured with a coarser equal area-grid. An iterative series expansion method was used. A good agreement between experiment and recalculation was found for the (002) pole figures, but the method also revealed the need to use more accurate measurement grids for a reliable determination of a complete ODF. A quantitative analysis of both measured and recalculated (002) pole figures was done to determine the volume fractions of texture components having a common preferred $c$-axis orientation. An error analysis was applied to these results. The effects of counting statistics and measurement grid were taken into account. For the latter, a simulation method based on data measured with a finer grid was used. The importance of a precise knowledge of the mean background was stressed. For its determination, a method based on fitting the total of the measured data with a Poisson distribution was proposed. However, when using this method, the possibility of a "fon" texture component of randomly oriented grains should be considered.

\section{Acknowledgements}

The authors would like to thank Dipl.-Phys. Min Zhi Wu and Dr. W. Gawalek from the IPHT Jena for the sample preparation (supported by the BMBF grant No. 13N6646), as well as Dr. H.-G. Brokmeier and his group at the GKSS Forschungszentrum for their help during the experiments.

The financial support of the BMBF (grant No. 13N6622) is gratefully acknowledged.

\section{References}

Bunge, H.-J. (1969). Mathematische Methoden der Texturanalyse. Berlin: Akademie Verlag. 
Bunge, H.-J. (1986). General Outline and Series Expansion Method. In Quantitative Texture Analysis, (ed.) H.-J. Bunge, 1-73. Oberursel: DGM Informationsgesellschaft m. b. H.

Chateigner, D., Wenk, H.-R. and Pernet, M. (1997). Orientation analysis of bulk YBCO from incomplete neutron diffraction data. Journal of Applied Crystallography, 30, 43-48.

Dahms, M. and Bunge, H.-J. (1989). The iterative series expansion method for quantitative texture analysis. I. General outline. Journal of Applied Crystallography, 22, 439-447.

Diko, P., Gawalek, W., Habisreuther, T., Klupsch, T. and Görnert, P. (1996). Macro- and microcracking, subgrains, twins and thermal stresses in $\mathrm{YBa}_{2} \mathrm{Cu}_{3} \mathrm{O}_{7-x}(123)$ $\mathrm{Y}_{2} \mathrm{BaCuO}_{5}(211)$ melt-textured superconductors studied by means of polarized light microscopy. Journal of Microscopy, 184, Pt. 1, 46-57.

Dimos, D., Chaudhari, P., Mannhart, J. and LeGoues, F.K. (1988). Orientation dependence of grain-boundary critical currents in $\mathrm{YBa}_{2} \mathrm{Cu}_{3} \mathrm{O}_{7-\delta}$ bicrystals. Physical Review Letters, 61, No. 2, 219-222.

Endo, A. and Shiohara, Y. (1997). Physica C, 276, 22-34.

Frangi, F., Jansak, L., Majoros, M. and Zannella, S. (1994). Trapped magnetic fields in melt-textured YBCO. Physica C, 224, 20-30.

Görnert, P. (1997). Crystal growth and crystalline layers of high temperature superconductors: characterization and application. Crystal Research and Technology, 32, 7-33.

Jin, S., Tiefel, T.H., Sherwood, M.E., Davis, R.B., van Dover, G.W., Kammlott, R.A. et al. (1988). Applied Physics Letters 52(24), 2074-2076.

JCPDS-ICDD PDF-2 database (1996). Card No. 39-486.

Marinel, S., Wang, J., Monot, I., Delamare, M.P., Provost, J. and Desgardin, G. (1997). Top-seeding melt texture growth of single-domain superconducting $\mathrm{YBa}_{2} \mathrm{Cu}_{3} \mathrm{O}_{7-\delta}$ pellets. Superconducting Science and Technology, 10, 147-155.

Sears, V.F. (1992). Scattering lengths for neutrons. In International Tables for Crystallography, Vol. C., 383-391. (Ed.) A. J. C. Wilson. Dordrecht: Kluwer Academic.

\section{APPENDIX}

\section{Derivation of Eq. (4)}

Inserting Eq. (3) into Eq. (2) yields:

$$
v_{i}=\sum_{j}^{\Omega_{i}}\left(I\left(\vec{y}_{j}\right)-\mathrm{BK}\right) \mathrm{d} \vec{y}_{j} / \sum_{k}^{2 \pi}\left(I\left(\vec{y}_{k}\right)-\mathbf{B K}\right) \mathrm{d} \vec{y}_{k},
$$

where $\sum_{j}^{\Omega_{i}}\left(I\left(\vec{y}_{j}\right)-\mathrm{BK}\right) \mathrm{d} \vec{y}_{j}$ is the integrated intensity of the area No. " $i$ ". In the following, it will be abbreviated by $\operatorname{Int}_{i}$. When $P_{\vec{h}}(\vec{y})$ is zero everywhere outside the various $\Omega_{i}$, then

$$
v_{i}=\operatorname{Int}_{i} / \sum_{k}^{\# \text { Areas }} \operatorname{Int}_{k} .
$$


When there is no overlap between various $\Omega_{i}$, then their integrated intensities are uncorrelated. Thus,

$$
\sigma_{\text {grid }}\left(v_{i}\right)=\left(\sum_{j}^{\# \text { Areas }}\left(\left(\partial v_{i} / \partial \operatorname{Int}_{j}\right) \sigma_{\text {grid }_{\text {gid }}}\left(\operatorname{Int}_{j}\right)\right)^{2}\right)^{1 / 2}
$$

with

$$
\begin{aligned}
& \partial v_{i} / \partial \operatorname{Int}_{j}=-\operatorname{Int}_{i} /\left(\sum_{k}^{\# \text { Areas }} \operatorname{Int}_{k}\right)^{2} \\
& =-v_{i} / \sum_{k}^{\# \text { Areas }} \operatorname{Int}_{k} \quad \text { for } i \neq j \text {, } \\
& \partial v_{i} / \partial \operatorname{Int}_{j}=\left(1-v_{i}\right) / \sum_{k}^{\# \text { Areas }} \operatorname{Int}_{k} \quad \text { for } i=j .
\end{aligned}
$$

Using the result of the simulation experiment, $\sigma_{\text {grid }}\left(\operatorname{Int}_{j}\right)=0.1 \operatorname{Int}_{j}($ and likewise for $i$ ):

$$
\begin{aligned}
& \sigma_{\text {grid }}\left(v_{i}\right)=0.1\left(\sum_{j \neq i}^{\# \text { Areas }}\left(\left(-v_{i}\right)^{2} \operatorname{Int}_{j}^{2} /\left(\sum_{k}^{\# \text { Areas }} \operatorname{Int}_{k}\right)^{2}\right)\right. \\
& \left.+\left(1-v_{i}\right)^{2} \operatorname{Int}_{i}^{2} /\left(\sum_{k}^{\# \text { Areas }} \operatorname{Int}_{k}\right)^{2}\right)^{1 / 2} . \\
& v_{j}=\operatorname{Int}_{j} / \sum_{k}^{\# \text { Areas }} \operatorname{Int}_{k} \text { and } v_{i}=\operatorname{Int}_{i} / \sum_{k}^{\# \text { Areas }} \operatorname{Int}_{k}
\end{aligned}
$$

then lead to Eq. (4). When there is more than one maximum in the integration area $\Omega_{j}$, then

$$
\sigma_{\text {grid }}\left(\operatorname{Int}_{j}\right)=0.1\left(\sum \operatorname{Int}_{\text {partial }}^{2}\right)^{1 / 2} \text {, with } \sum \operatorname{Int}_{\text {partial }}=\operatorname{Int}_{j},
$$

where the sum is over the number of partial maxima in $\Omega_{j}$. Then, $\sigma_{\text {grid }}\left(\operatorname{Int}_{j}\right)<0.1 \operatorname{Int}_{j}$ because of $\operatorname{Int}_{\text {partial }}>0 . \sigma_{\text {grid }}\left(v_{j}\right)$ will then also be smaller. 


\section{Derivation of Eq. (5)}

The derivative of

$$
v_{i}=\sum_{j}^{\Omega_{i}}\left(I\left(\vec{y}_{j}\right)-\mathrm{BK}\right) \mathrm{d} \vec{y}_{j} / \sum_{k}^{2 \pi}\left(I\left(\vec{y}_{k}\right)-\mathrm{BK}\right) \mathrm{d} \vec{y}_{k}
$$

with respect to BK yields

$$
\begin{aligned}
\partial v_{i} / \partial \mathrm{BK}= & \left(-\sum_{j}^{\Omega_{i}} \mathrm{~d} \vec{y}_{j}\right) / \sum_{k}^{2 \pi}\left(I\left(\vec{y}_{k}\right)-\mathrm{BK}\right) \mathrm{d} \vec{y}_{k} \\
& +v_{i}\left(\sum_{j}^{2 \pi} \mathrm{d} \vec{y}_{j}\right) / \sum_{k}^{2 \pi}\left(I\left(\vec{y}_{k}\right)-\mathrm{BK}\right) \mathrm{d} \vec{y}_{k} .
\end{aligned}
$$

Defining $\langle I\rangle$ as: $\sum_{k}^{2 \pi} I\left(\vec{y}_{k}\right) \mathrm{d} \vec{y}_{k} /(2 \pi)$ yields

$$
\begin{aligned}
\partial v_{i} / \partial \mathrm{BK} & =\left(-\Omega_{i}+2 \pi v_{i}\right) /(2 \pi(\langle I\rangle-\mathrm{BK})), \\
& =2 \pi v_{i}\left(1-\Omega_{i} /\left(2 \pi v_{i}\right)\right) /(2 \pi(\langle I\rangle-\mathrm{BK})) .
\end{aligned}
$$

When all other variables (i.e. the $I\left(\vec{y}_{k}\right)$ ) are kept constant, then $\Delta v_{i}=$ $\left(\partial v_{i} / \partial \mathrm{BK}\right) \Delta \mathrm{BK}$, where $\Delta \mathrm{BK}$ is a small increment of $\mathrm{BK}$.

Note: Changing BK while keeping the measured intensities $I\left(\vec{y}_{k}\right)$ constant means that the estimated contribution of the background to the $I\left(\vec{y}_{k}\right)$ is changed.

Factorising BK from the denominator of $\partial v_{i} / \partial \mathrm{BK}$ then leads to Eq. (5). 